\title{
Efficacy of relaxation therapy as an effective nursing intervention for post-operative pain relief in patients undergoing abdominal surgery: A systematic review and meta-analysis
}

\author{
WANXIA JU, LILI REN, JUN CHEN and YUMAN DU \\ Department of General Surgery, The Fifth Central Hospital of Tianjin, Tianjin 300450, P.R. China
}

Received July 6, 2019; Accepted August 9, 2019

DOI: 10.3892/etm.2019.7915

\begin{abstract}
This systematic review and meta-analysis was conducted with the aim of assessing the efficacy of relaxation techniques for pain relief in patients undergoing abdominal surgery. The electronic search of the PubMed, Scopus, Cochrane Central Register of Controlled Trials (CENTRAL) and Google Scholar databases was performed for studies in the English language published up to May, 2019. A total of 12 studies were included in the review and 7 in the meta-analysis. In total, 4 relaxation techniques were utilized in the included studies: Jaw relaxation, Benson's relaxation, progressive muscle relaxation (PMR) and systematic relaxation. Of the 12 included, 10 studies demonstrated statistically significant pain relief in the relaxation group as compared to the controls. The data of 422 patients in the relaxation group and 424 patients in the control group were pooled for a meta-analysis, which indicated that patients undergoing abdominal surgery had significantly greater pain relief following relaxation therapy as compared to the controls [random: standardized mean difference (SMD), -1.15 ; $95 \% \mathrm{CI},-2.04$ to -0.26 ; $\mathrm{P}<0.00001)$. The overall quality of the studies was not high. On the whole, despite trials demonstrating the benefits of relaxation therapy for immediate pain relief in patients post-abdominal surgery, there is lack of high-quality scientific evidence substantiating its routine use. There is a need for more robust randomized control trials (RCTs) utilizing standardized relaxation protocols to provide further evidence on this subject.
\end{abstract}

\section{Introduction}

The International Association for the Study of Pain, describes pain as 'an unpleasant sensory and emotional experience associated with actual or potential tissue damage or described in terms of such damage' (1). A large majority of patients

Correspondence to: Dr Wanxia Ju, Department of General Surgery, The Fifth Central Hospital of Tianjin, 41 Zhejiang Road, Tianjin 300450, P.R. China

E-mail: jwx.xin@qq.com

Key words: relaxation techniques, surgery, pain, nursing care undergoing surgery experience post-operative pain, which is not only agonizing and distressing, but may also contribute to complications and to a delayed recovery (2). Pain exacerbates stress responses, which lead to increased tissue breakdown, coagulation and fluid retention, and have deleterious effects on the recuperation of patients (3).

Abdominal surgery is considered to be one of the most painful surgical procedures (4). The site's proximity to the diaphragm and extensive cross-innervations in the area multiply the post-operative distress experienced by patients (5). Inadequate pain control in such individuals can lead to impaired respiration, disrupted sleep, loss of appetite, prolonged hospitalization, patient dissatisfaction and increased treatment costs $(4,6,7)$.

Pain management has been a subject of intense research with a number of technological advancements striving to achieve optimal pain control. Even with the advent of patient- controlled analgesia, continuous intravenous infusion and the intraspinal application of opioids, pain control remains a major challenge (4). Post-operative patients are under the constant supervision of nurses, who also are responsible for assessing the need and type of pain relief (3). While the administration of analgesics is an important part of nursing practice, non-pharmacological interventions for the reduction of post-operative pain are slowly gaining popularity. Patient education, massages, music, guided imagery and relaxation therapy are being increasingly used by nurses in the post-operative setting to achieve more effective pain relief (8).

Relaxation therapy has been shown to provide pain relief by decreasing anxiety, lowering muscle tension and distracting attention (9). While a number of studies $(4,7,9)$ have described the beneficial effects of relaxation therapy for pain relief in patients undergoing abdominal surgery, evidence in the form of a systematic review and meta-analysis is lacking, at least to the best of our knowledge. Therefore, the aim of this study was to perform a systematic literature search and critically analyze available evidence with the objective of assessing the efficacy of relaxation techniques for pain relief in patients undergoing abdominal surgery.

\section{Data and methods}

This systematic review of the literature was conducted in line with the Preferred Reporting Items for Systematic Reviews 
and Meta-analyses (PRISMA) statement (10) and guidelines of the Cochrane Handbook for Systematic Reviews of Intervention (11). The study protocol was prepared prior to the commencement of the study. The issue in question was the effectiveness of relaxation techniques against post-operative pain in patients undergoing abdominal surgery.

Eligibility criteria. We searched for studies evaluating the effects of relaxation therapy in patients undergoing abdominal surgery. No restrictions were placed on the type of relaxation technique and the type of abdominal surgery. Controlled clinical trials (CCTs) and randomized control trials (RCTs) studying the effects of relaxation therapy on post-operative pain reduction in patients undergoing abdominal surgery were included in this review. The participants of the included studies needed to be $>18$ years of age, had to have undergone surgery under general anesthesia or spinal anesthesia and must have been hospitalized during the period of the surgery for at least 48 h. Participants were not to have any cognitive impairment and were not to have planned to undergo any neurosurgery. Intervention had to include relaxation therapy with muscle relaxation in the post-operative period. Outcome assessment had to include pain scores measured on any scale, such as the visual analogue scale (VAS) (3). The studies excluded were the following: i) Studies combining relaxation therapy with other psychological pain relief measures, such as guided imagery; ii) studies in which relaxation therapy was performed pre-operatively; and iii) studies which did not include pain as an outcome variable. Additionally, non-English language studies, studies on healthy volunteers and uncontrolled studies were also excluded.

Search strategy. We searched the PubMed, Scopus, Cochrane Central Register of Controlled Trials (CENTRAL) and Google Scholar databases (first 100 results) electronically for articles published up to May, 2019. The key words used in various combinations were: Relaxation therapy [MeSH], relaxation techniques $[\mathrm{MeSH}$, progressive muscle relaxation [Free text], systematic relaxation [Free text], Benson's relaxation [Free text], abdominal surgery [Free text], surgery [MeSH], pain [MeSH], pain relief [MeSH], abdominal pain [MeSH], nursing $[\mathrm{MeSH}]$ and nursing care [MeSH]. References of included studies and review articles were analysed for the identification of any additional studies.

Collection of data and analysis. Two reviewers examined potentially eligible studies. Following the removal of duplicates, studies were scrutinized by their title and abstracts. Full-texts of selected articles were then scanned for their inclusion in the review. Any differences in opinion were resolved by discussion. The following data were extracted from the included trials: Authors, publication year, sample size, demographic data, relaxation technique, relaxation protocol and outcomes assessed.

Evaluating risk of bias. The Cochrane Collaboration risk assessment tool for RCTs was used for assessing the risk of bias (12). Seven criteria were evaluated for each study: Random sequence generation, allocation concealment, blinding of participants and personnel, blinding of outcome assessment, incomplete outcome data, selective outcome reporting and other biases. Studies were scored for each criteria as follows: Low risk (score of 2), high risk (score of 0 ), or unclear risk of bias (score of 1). Based on the scores awarded, individual studies were grouped as low- (score 0-5), medium- (score 6-10), or high- (score 11-14) quality trials.

Statistical analysis. Studies were included in the meta-analysis only if pain scores were reported as the means and standard deviation. The results of the remaining studies were summarized in a narrative form. Outcome data extracted were entered into Review Manager [RevMan, version 5.3; Nordic Cochrane Centre (Cochrane Collaboration), 2014] for quantitative analysis. the data of different relaxation techniques were pooled for a sub-group analysis. Considering the heterogeneity amongst studies, a random-effects model was used to calculate the pooled effect size. The standardized mean difference (SMD) with the $95 \%$ confidence interval (CI) was used for combining pain data measured on different scales. Heterogeneity was calculated using the $\mathrm{I}^{2}$ statistic. $\mathrm{I}^{2}$ values of $25-50 \%$ represented low, values of $50-75 \%$ medium and $>75 \%$ represented substantial heterogeneity.

\section{Results}

Search outcome. The search outcome of the review is presented in Fig. 1. A total of 1,092 articles were examined by their abstracts. We excluded a total of 1,072 studies as they were not relevant to the study. In total, 20 articles were analyzed by their full text. Seven studies were excluded from the review (13-19). Two studies did not include pain as an outcome measure (15,18), 1 did not include a control group (19), 1 was a duplicate publication (13), the relaxation technique did not include actual muscle relaxation in 1 study (17), 1 combined guided imagery with relaxation therapy (16) and 1 administered relaxation therapy only pre-operatively (14). A total of 12 studies $(2,4,7,9,20-27)$ were included in the qualitative review and 7 trials $(2,7,9,21,23,24,27)$ were included in the meta-analysis.

Relaxation techniques. A total of 4 relaxation techniques were utilized in the included studies, namely: The jaw relaxation technique, Benson's relaxation technique, progressive muscle relaxation (PMR) technique and systematic relaxation technique. The description of each technique was as follows: i) The jaw relaxation technique was carried out with patients dropping the lower jaw slightly as though starting a small yawn. The tongue was kept quiet and resting at the bottom of the mouth. The lips were to parted slightly, and the patient was to breath slowly and rhythmically with a 3-rhythm pattern (inhale, exhale and rest). Word formation was not advised and the patient was advised to not think of any words $(9,27)$. ii) Benson's relaxation therapy involved deep relaxation of all muscles, focusing on breathing and repeating any word to focus on relaxation, and preventing the wandering of the individual's attention $(20,24)$. iii) PMR consisted of deep breathing along with the systemic sequential tensing of the muscles $(5-7 \mathrm{sec})$ followed by relaxation $(10-12 \mathrm{sec})$. The technique was repeated for different muscle groups until the entire body was relaxed $(21-23,25)$. iv) Systematic relaxation differed 


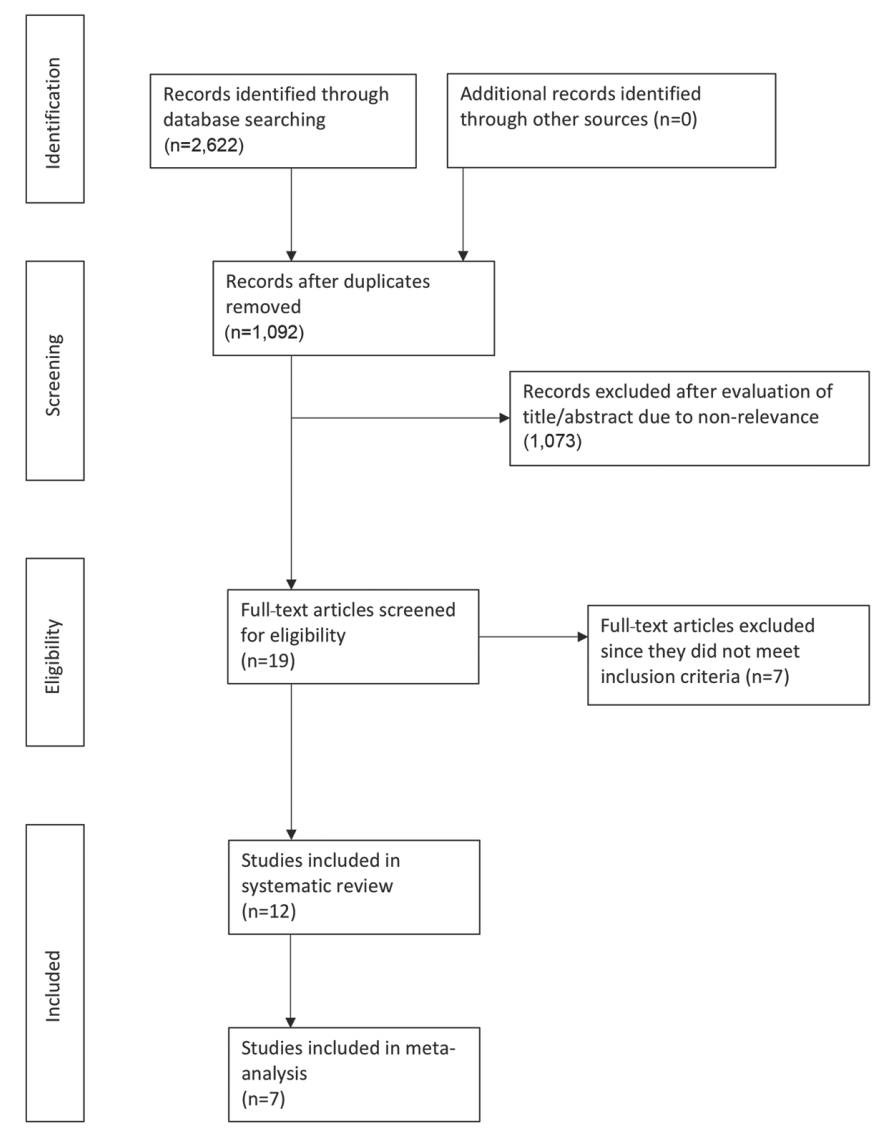

Figure 1. Flowchart of the study.

from PMR as no tensing of the muscles was carried out in this technique and focus was only placed on sequentially relaxing individual groups of muscles $(2,7,26)$.

Characteristics of included studies. The details of the included studies are presented in Table I. The sample sizes of the included studies varied from a minimum of 17 patients per group to a maximum of 130 patients. The studies included a combination of patients undergoing gastrointestinal or gynecological surgical procedures. PMR was the most commonly studied relaxation therapy with 4 trials reporting its use $(21-23,25)$. Systematic relaxation was studied by 3 trials $(2,7,26)$. There were 2 studies each for jaw relaxation $(9,27)$ and Benson's relaxation therapy $(20,24)$, and 1 trial (4) did not specify the type of relaxation technique used. The relaxation technique were explained to the interventional group pre-operatively in all studies and practiced for use in the post-operative period. Considerable variation was observed in the relaxation protocol used across studies. Relaxation therapy was practiced at a specific time in all studies apart from 2. In the studies by Wilson (26) and Levin et al (20), relaxation was performed by the patients as and when required. Pain scores were measured just after the completion of the relaxation therapy in all studies, apart from 2. The study by Devmurari and Nagrale (22) compared pain scores after the completion of 5 days of PMR therapy, while the study by Ismail and Elgzar (21) compared pain scores after 2 days of therapy. In total, 10 of the 12 included studies demonstrated statistically significant pain relief in the relaxation group as compared to controls $(2,4,7,9,20,21,23,25-27)$. The study by Solehati and Rustina (24) utilizing Benson's relaxation technique found significant pain relief in both the interventional and control group. The study by Devmurari and Nagrale (22) reported greater pain reduction in the PMR group; however, no statistical analysis was carried out.

Meta-analysis. Seven of the 12 included studies reported sufficient data for inclusion in the meta-analysis $(2,7,9,21,23,24,27)$. The data of 422 patients in the relaxation group and 424 patients in the control group were pooled for a quantitative analysis. The results indicated that patients undergoing abdominal surgery experienced significantly greater pain relief following relaxation therapy as compared to the controls (random: SMD, $-1.15 ; 95 \%$ CI, -2.04 to -0.26 ; $\mathrm{P}<0.00001$ ) (Fig. 2). There was significant heterogeneity amongst studies with $\mathrm{I}^{2}=96 \%$. Sub-group analysis demonstrated statistically significant pain relief in patients undergoing abdominal surgery and performing jaw relaxation (random: SMD, -0.50; 95\% CI, -1.2 to $0.2 ; \mathrm{P}=0.03$ ), systematic relaxation (random: $\mathrm{SMD},-1.7$; 95\% CI, -3.42 to $0.01 ; \mathrm{P}<0.00001$ ); and PMR (random: SMD, $-2.06 ; 95 \%$ CI, -6.19 to 2.07 ; $\mathrm{P}<0.00001)$.

Outcome variables other than pain. A number of additional outcome variables were compared between the relaxation and control groups by the included studies. Systemic measures, such as pulse rate, blood pressure and respiratory rate were analyzed by 3 studies $(9,25,27)$. Flaherty and Fitzpatrick (27), in their study on jaw relaxation, recorded vital signs of the 2 groups at the point when pain was being measured. There was no significant difference in pulse rates and blood pressure between the 2 groups. However, they found a greater post-operative decrease in the respiratory rate in the relaxation group. Similarly, Good et al (9), in their study on jaw relaxation therapy, found significant decrease in pulse rates and respiratory rates in the interventional group. On the other hand, Chandrababu (25), in a trial on PMR, reported no effect of relaxation therapy on respiratory rates and blood pressure.

Studies on systematic relaxation reported conflicting results on the effects of relaxation on post-operative anxiety. While Roykulcharoen and Good (2) demonstrated no difference in post-operative anxiety between the 2 groups, Rejeh et al (7) recorded significantly less anxiety in the relaxation group. Similarly, while the studies by Levin (20) and Roykulcharoen and Good (2) found no difference in analgesic consumption between the relaxation and control groups, Rejeh et al (7) recorded significantly less analgesic use in the group practicing systematic relaxation.

Quality of included studies. The risk of bias summary of the included studies is presented in Fig. 3. Only 5 trials reported an adequate information on the randomization method used $(7,9,20-22)$. Blinding was reported by only 2 of the 12 studies $(9,26)$. Based on the scoring criteria, there was only 1 high quality study, while all others were rated as 'medium'.

\section{Discussion}

As shown by our literature review, relaxation therapy has been a subject of substantial research in the area of pain relief 


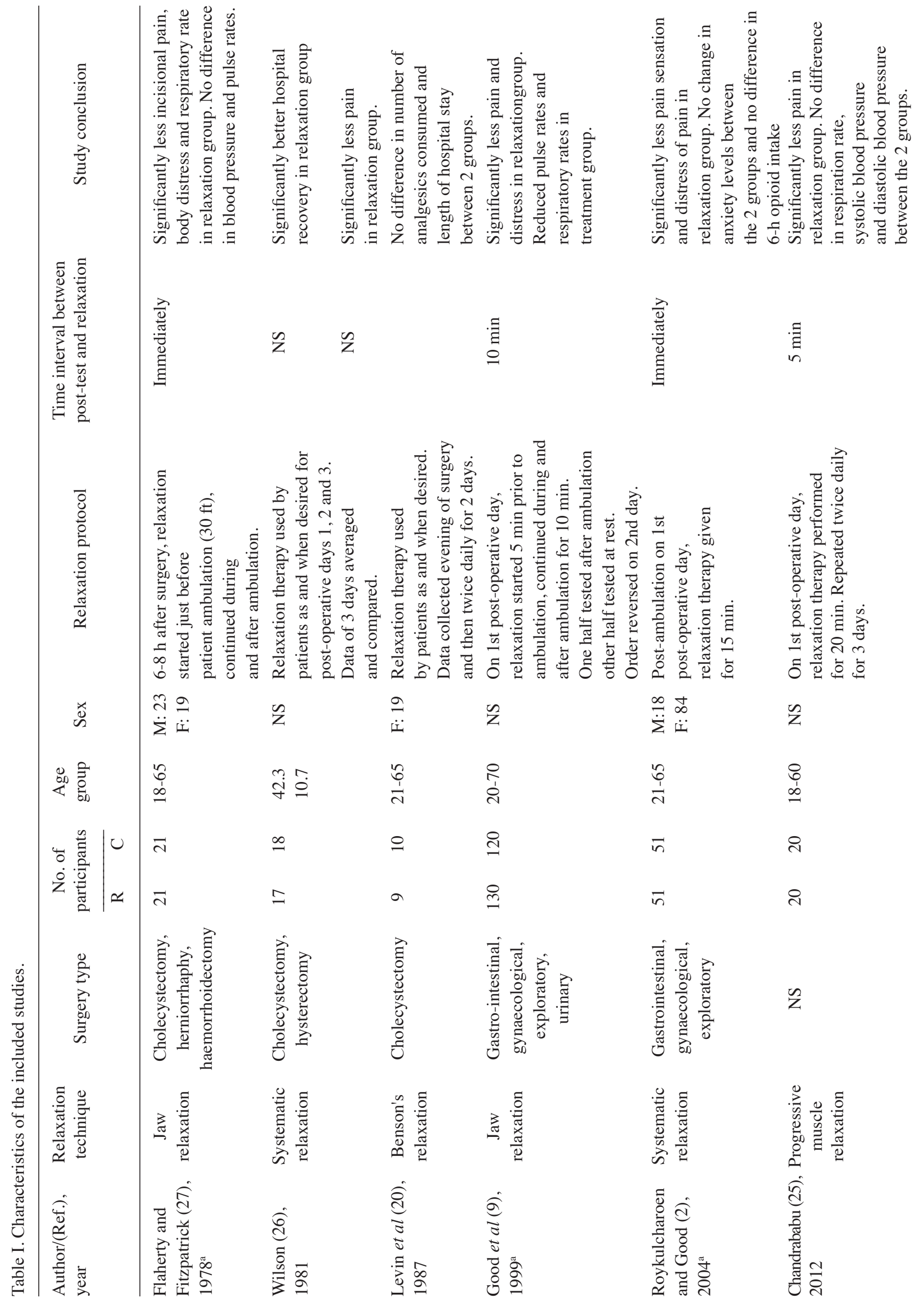




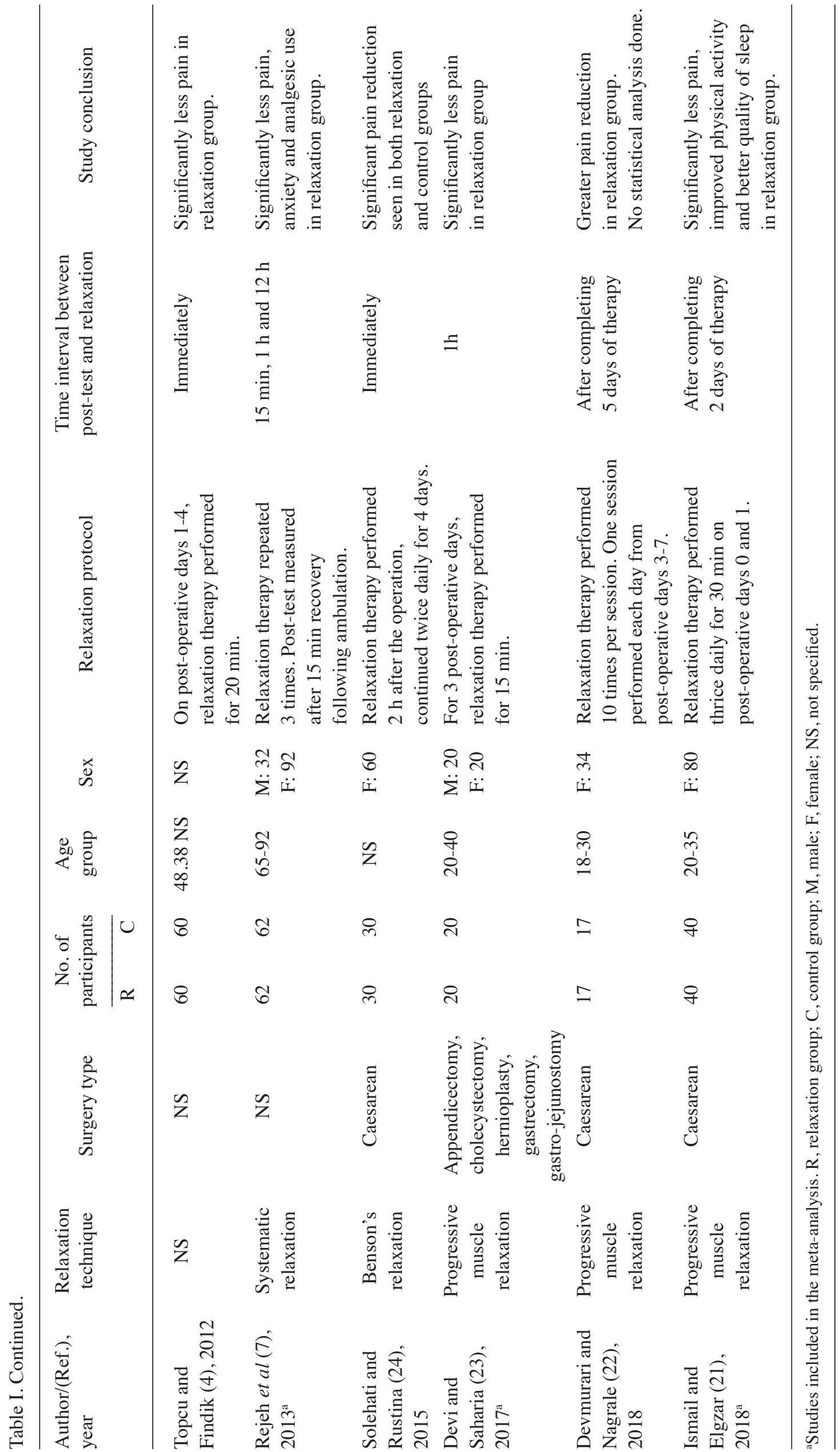




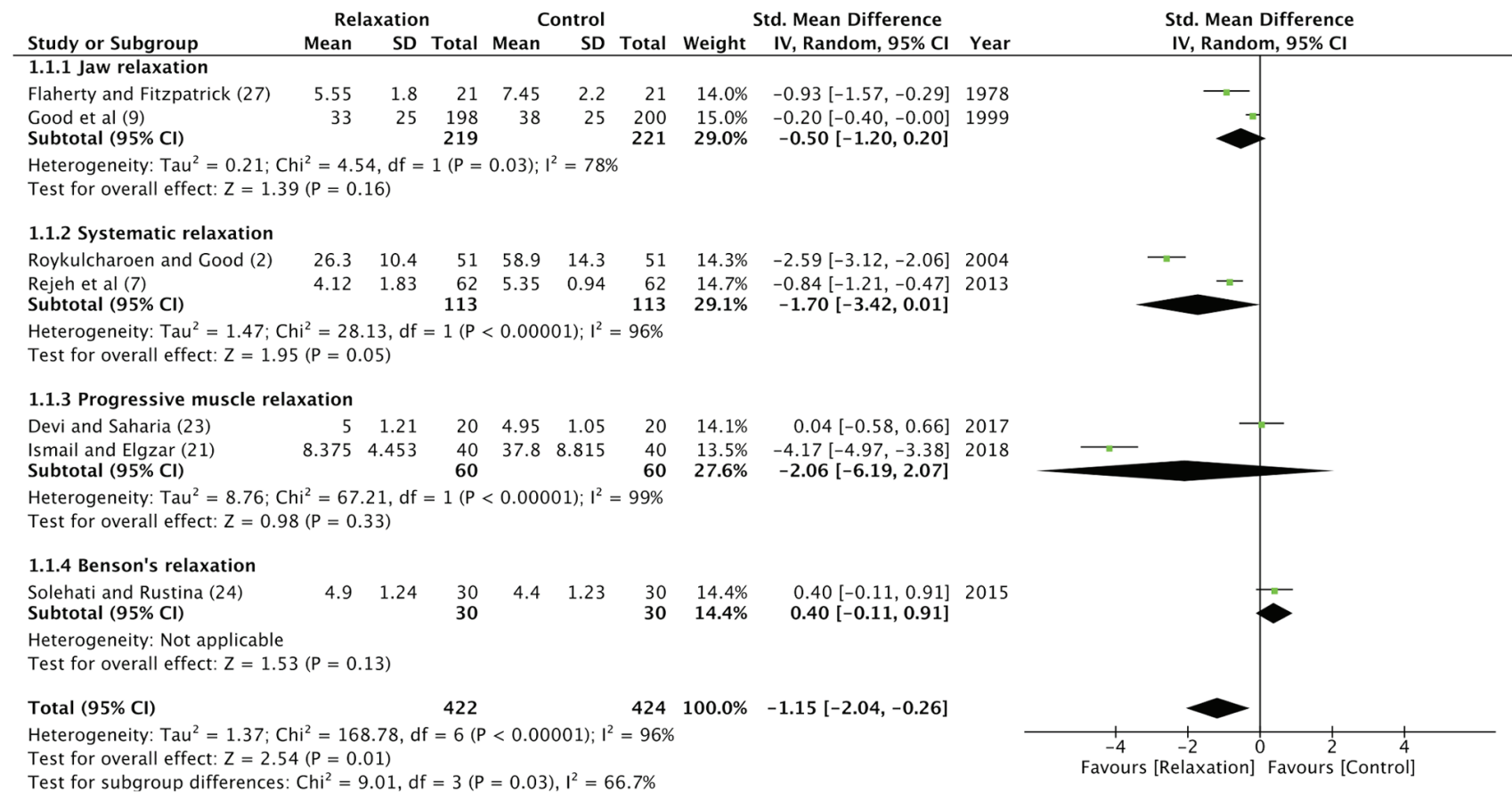

Figure 2. Forrest plot of relaxation therapy for pain relief. CI, confidence interval.

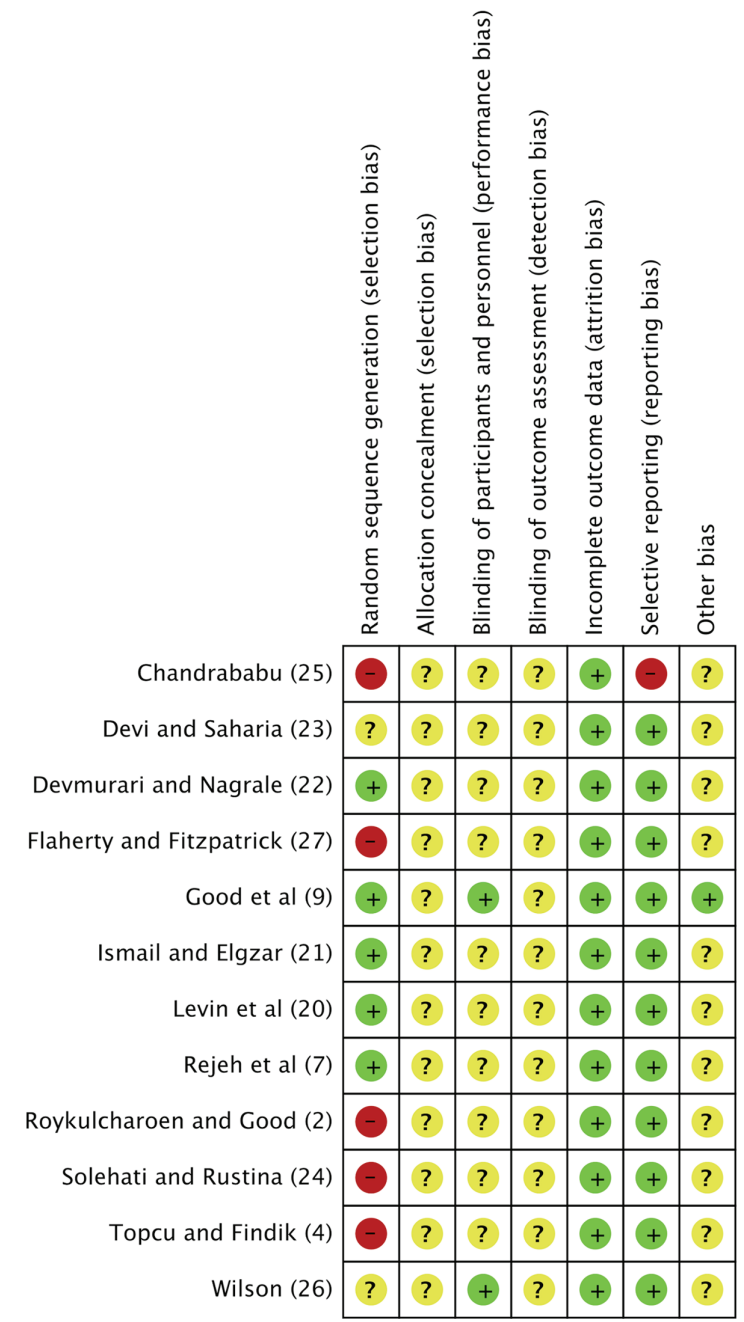

Figure 3. Risk of bias summary (green circles indicate a low risk of bias, yellow circles indicate an unclear risk of bias and red circles indicate a high risk of bias). after abdominal surgery. Studies have compared 4 different relaxation methods to date. 'Jaw relaxation', which involves the relaxation of the mouth and throat, is a specific regional approach developed by Jacobson $(9,28)$. He proposed that as skills in releasing tension in the local parts of the body are developed, the relaxation of the entire body becomes easier. This technique was investigated by two of the included studies in this review. Flaherty and Fitzpatrick (27) and Good et al (9) both demonstrated significant difference in pain relief between patients practicing jaw relaxation therapy and controls. Similar results with jaw relaxation have been replicated by other researchers in the field of orthopedic surgery $(28,29)$. Ceccio et al (28) studied post-movement pain, in the first $24 \mathrm{~h}$ following hip-surgery in elderly patients. They reported significantly less pain in the group practicing jaw relaxation. Seers et al (29) found statistically significant reduction in pain with both jaw relaxation and total body relaxation in patients undergoing elective orthopedic surgery.

Total body relaxation techniques used by studies in our review included, Benson's relaxation, PMR and systematic relaxation. Only minor differences separate these 3 techniques (2). Systematic relaxation differs from PMR, as there is no muscle contraction involved; which according to some authors may be painful in a post-operative surgical patient (2). Benson's relaxation and systematic relaxation are quite similar apart from the fact that participants are more focused on breathing and the repetition of a single word in Benson's technique. Upon the pooled analysis of all the studies, we found that patients utilizing relaxation techniques experienced a statistically significant reduction in pain, as compared to individuals receiving usual nursing care. These results were also significant for individual sub-groups of jaw relaxation, PMR and systematic relaxation. While our review focused only on abdominal surgery patients, relaxation techniques have been used as a non-pharmacological 
pain relief intervention in a number of other disorders. De Paolis et al (30) found PMR with interactive imagery to be an effective adjuvant pain relief technique for terminal cancer patients. Jong et al (31) used PMR as an effective tool for pain relief in children with primary headaches.

The mechanism of pain relief with relaxation therapy has been explained in relation to the gate control theory of pain. The gate control theory of pain postulates that alteration or modification of pain impulses being transmitted from the peripheral nerve receptors to the brain can result in little or no pain perception (32). Substansia gelatinosa, a group of densely packed cells along the length of the spinal cord, are considered the site of transmission blockage. When 'open', this area allows for the transmission of pain sensation to reach the dorsal horn of the spinal cord. Emotional and cognitive factors are thought to influence this sensory transmission $(27,28)$. Fear, anxiety, attention to pain, past experiences etc., all influence the pain response by acting on the gate control system (33). Relaxation therapy is thought to affect this cognitive and emotional alteration thereby providing pain relief. Melzack and Chapman (34) suggested that the distraction of attention from the painful site, the reduction of anxiety and the development of a sense of control over pain by means of relaxation therapy can help diminish the noxious feeling. Relaxation also helps diminish muscular and mental tension thereby reducing sympathetic stimulation of the hypothalamus. This modulates endogenous opioid production in the nervous systems which in turn decreases propagation of pain impulses (35-37).

The actual effect of relaxation therapy on anxiety, stress and systemic variables (pulse, blood pressure and respiratory rate) in abdominal surgery have also been studied. While some studies $(7,18)$ have report significant reductions in post-operative anxiety with relaxation therapy, others differ (2). Similar variable results have been reported for pulse rate, respiratory rate and blood pressure $(9,15,25,27)$. Post-operative stress in patients with colorectal cancer has been estimated using serum cortisol levels by Kim et al (15). Significant differences were found between relaxation and control group on the first post-operative day. The conflicting results amongst studies may be attributed to a number of factors, such as small sample sizes in trials demonstrating significant results (18), variable relaxation protocols, difference in anxiety scales used, errors is timing and measurement methods for systemic variables etc.

The strength of any meta-analysis to a certain extent depends on the homogeneity and quality of the studies included. A number of factors limit our reviews ability to draw strong conclusions for relaxation therapy. Foremost, there was only one high quality study (9) available for inclusion. The methodology of the remaining studies was questionable with absence of proper randomization and the blinding of outcome assessment. Secondly, only 1 study included $>100$ participants per group (9). A number of trials were underpowered with $<20$ patients in each group $(20,22,26)$. Thirdly, the was a wide variation in the relaxation protocol used, with studies employing the therapy at different times and for varying periods of time. There was disparity in the pain scales used, with both 10-point and 100-mm scales of VAS utilized across studies. Fourthly, there was no longterm data available on the effects of relaxation therapy on post-operative pain relief. Seers et al (29) demonstrated that pain relief following relaxation was not sustained for a long period of time. While the majority of studies measured pain immediately following relaxation therapy and found significant differences between the intervention and control groups, the study by Devmurari and Nagrale (22), while comparing pain scores in post-caesarean females, after 5 days of relaxation therapy, they found a significant reduction in pain in both the interventional and control groups.

Despite a number of trials demonstrating benefits of relaxation therapy for immediate pain relief in patients undergoing post-abdominal surgery, there is lack of high-quality scientific evidence substantiating its routine use. There is a need for more robust RCTs utilizing standardized relaxation protocols to provide further evidence on this subject. However, in the absence of harmful effects of relaxation therapy and minimal time required for training patients, despite weak evidence, it may still be employed by nurses in the post-operative setting to provide short-term pain relief.

\section{Acknowledgements}

Not applicable.

\section{Funding}

No funding was received.

\section{Availability of data and materials}

The datasets used and/or analyzed during the current study are available from the corresponding author on reasonable request.

\section{Authors' contributions}

WJ conceived and designed the study. LR, JC and YD collected the data and performed the literature search. All authors were involved in the writing of the manuscript. All authors have read and approved the final manuscript.

\section{Ethics approval and consent to participate}

Not applicable.

\section{Patient consent for publication}

Not applicable.

\section{Competing interests}

The authors declare that they have no competing interests.

\section{References}

1. IASP pain terminology: International Association for the Study of Pain (IASP), 2010.

2. Roykulcharoen V and Good M: Systematic relaxation to relieve postoperative pain. J Adv Nurs 48: 140-148, 2004.

3. Miaskowski C: Current concepts in the assessment and management of acute pain. Medsurg nursing: official journal of the Academy of Medical-Surgical Nurses 2: 28-32, 40, 1993.

4. Topcu SY and Findik UY: Effect of relaxation exercises on controlling postoperative pain. Pain Manag Nurs 13: 11-17, 2012. 
5. Jurf JB and Nirschl AL: Acute postoperative pain management: A comprehensive review and update. Crit Care Nurs Q 16: 8-25, 1993.

6. Shang $A B$ and Gan TJ: Optimising postoperative pain management in the ambulatory patient. Drugs 63: 855-867, 2003

7. Rejeh N, Heravi-Karimooi M, Vaismoradi M and Jasper M: Effect of systematic relaxation techniques on anxiety and pain in older patients undergoing abdominal surgery. Int J Nurs Pract 19 : 462-470, 2013

8. Crowe L, Chang A, Fraser JA, Gaskill D, Nash R and Wallace K: Systematic review of the effectiveness of nursing interventions in reducing or relieving post-operative pain. JBI Library Syst Rev 6: 165-224, 2008.

9. Good M, Stanton-Hicks M, Grass JA, Cranston Anderson G, Choi C, Schoolmeesters LJ and Salman A: Relief of postoperative pain with jaw relaxation, music and their combination. Pain 81: 163-172, 1999.

10. Moher D, Liberati A, Tetzlaff J and Altman DG; PRISMA Group: Preferred reporting items for systematic reviews and meta-analyses: The PRISMA statement. PLoS Med 6: e1000097, 2009.

11. Higgins JPT and Green S: Cochrane Handbook for Systemic Reviews of Interventions. Version 5. The Cochrane Collaboration, 2011.

12. Higgins JPT, Altman D and Sterne J; Cochrane Statistical Methods Group and the Cochrane Bias Methods Group: Chapter 8: Assessing risk of bias in included studies. In: Cochrane Handbook for Systemic Reviews of Interventions. Higgins JPT and Green S(eds). Version 5. The Cochrane Collaboration, 2011

13. Good M, Anderson GC, Ahn S, Cong X and Stanton-Hicks M Relaxation and music reduce pain following intestinal surgery. Res Nurs Health 28: 240-251, 2005.

14. Hasanpour-Dehkordi A, Solati K, Tali SS and Dayani MA: Effect of progressive muscle relaxation with analgesic on anxiety status and pain in surgical patients. Br J Nurs 28: 174-178, 2019.

15. Kim KJ, Na YK and Hong HS: Effects of progressive muscle relaxation therapy in colorectal cancer patients. West J Nurs Res 38: 959-973, 2016.

16. Broadbent E, Kahokehr A, Booth RJ, Thomas J, Windsor JA Buchanan CM, Wheeler BR, Sammour T and Hill AG: A brief relaxation intervention reduces stress and improves surgical wound healing response: A randomised trial. Brain Behav Immun 26: 212-217, 2012

17. Haase O, Schwenk W, Hermann C and Müller JM: Guided imagery and relaxation in conventional colorectal resections: A randomized, controlled, partially blinded trial. Dis Colon Rectum 48: 1955-1963, 2005.

18. Cheung YL, Molassiotis A and Chang AM: A pilot study on the effect of progressive muscle relaxation training of patients after stoma surgery. Eur J Cancer Care (Engl) 10: 107-114, 2001.

19. de Paula AAD, de Carvalho EC and dos Santos CB: The use of the 'progressive muscle relaxation' technique for pain relief in gynecology and obstetrics. Rev Lat Am Enfermagem 10 654-659, 2002

20. Levin RF, Malloy GB and Hyman RB: Nursing management of postoperative pain: Use of relaxation techniques with female cholecystectomy patients. J Adv Nurs 12: 463-472, 1987.
21. Aziz Ismail NIA and Elgzar WTI: The effect of progressive muscle relaxation on post cesarean section pain, quality of sleep and physical activities limitation. Int J Stud Nurs 3: 14-29, 2018.

22. Devmurari D and Nagrale S: Effectiveness of Jacobson's progressive muscle relaxation technique for pain management in post-cesaerean women. Indian J Obstet Gynecol Res 5: 228-232, 2018.

23. Devi R and Saharia H: Effect of progressive muscle relaxation on postoperative analgesia. Indian J Med Res Rev 5: 113-118, 2017.

24. Solehati T and Rustina Y: Benson relaxation technique in reducing pain intensity in women after cesarean section. Anesth Pain Med 5: e22236, 2015.

25. Chandrababu R: Effectiveness of progressive muscle relaxation technique on pain perception among patients who are subjected to abdominal surgery. Indian J Surg Nurs 1: 83-88, 2012.

26. Wilson JF: Behavioral preparation for surgery: Benefit or harm? J Behav Med 4: 79-102, 1981

27. Flaherty GG and Fitzpatrick JJ: Relaxation technique to increase comfort level of postoperative patients: A preliminary study. Nurs Res 27: 352-355, 1978.

28. Ceccio CM: Postoperative pain relief through relaxation in elderly patients with fractured hips. Orthop Nurs 3: 11-19, 1984.

29. Seers K, Crichton N, Tutton L, Smith L and Saunders T: Effectiveness of relaxation for postoperative pain and anxiety: Randomized controlled trial. J Adv Nurs 62: 681-688, 2008.

30. De Paolis G, Naccarato A, Cibelli F, D'Alete A, Mastroianni C, Surdo L, Casale G and Magnani C: The effectiveness of progressive muscle relaxation and interactive guided imagery as a pain-reducing intervention in advanced cancer patients: A multicentre randomised controlled non-pharmacological trial. Complement Ther Clin Pract 34: 280-287, 2019.

31. Jong MC, Boers I, van Wietmarschen HA, Tromp E, Busari JO, Wennekes R, Snoeck I, Bekhof J and Vlieger AM: Hypnotherapy or transcendental meditation versus progressive muscle relaxation exercises in the treatment of children with primary headaches: A multi-centre, pragmatic, randomised clinical study. Eur J Pediatr 178: 147-154, 2019.

32. Davis P: Pain: Opening up the gate control theory. Nurs Stand 7: 25-27, 1993.

33. Casey KL: The neurophysiologic basis of pain. Postgrad Med 53: 58-63, 1973.

34. Melzack R and Chapman CR: Psychologic aspects of pain. Postgrad Med 53: 69-75, 1973.

35. Vidal C and Jacob J: The effect of medial hypothalamus lesions on pain control. Brain Res 199: 89-100, 1980

36. Standley JM: Music research in medical/dental treatment: Meta-analysis and clinical applications. J Music Ther 23: 56-122, 1986.

37. Turner JA and Chapman CR: Psychological interventions for chronic pain: A critical review. II. Operant conditioning, hypnosis, and cognitive-behavioral therapy. Pain 12: 23-46, 1982.

This work is licensed under a Creative Commons Attribution-NonCommercial-NoDerivatives 4.0 International (CC BY-NC-ND 4.0) License. 